\title{
A Local DCT-II Feature Extraction Approach for Personal Identification Based on Palmprint
}

\author{
H. Kipsang Choge Non-member (The University of Tokushima) \\ Tadahiro Oyama Non-member (Kobe City College of Technology) \\ Stephen Karungaru Member (The University of Tokushima) \\ Satoru Tsuge Member (Daido University) \\ Minoru Fukumi Member (The University of Tokushima)
}

Keywords: palmprint recognition, local DCT, biometric feature extraction, hand-based biometrics

Biometric applications based on the palmprint have recently attracted increased attention because they are easy to use and have a high user acceptance due to their non-invasive nature.

This paper presents a holistic method that makes use of the Discrete Cosine Transform (DCT) in a novel way by exploiting its data-compacting strengths while eliminating or minimizing the weak points through careful preprocessing of the input palmprints. Position and brightness normalization is achieved using the steps summarized in Fig. 1. The segmented region of interest (ROI) is divided into blocks of either $8 \times 8$ or $16 \times 16$ pixels in size before applying the type-II DCT on them. A subset of coefficients that encode the low to medium frequency components is selected using the JPEGstyle zigzag scanning method. Features from each block are then concatenated into a feature vector and used in various palmprint verification and recognition experiments with palmprints from the 'PolyU' Palmprint Database to test the

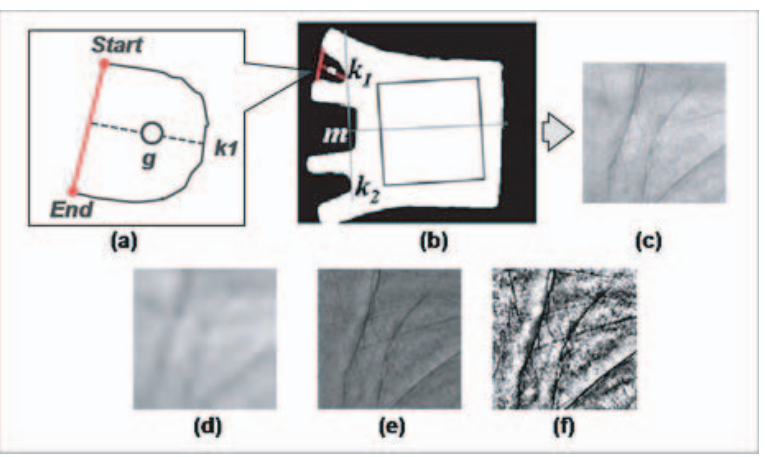

Fig. 1. The preprocessing steps used for extraction and normalization of the ROI: (a) starting and end points for calculating center of gravity, $g$, (b) the 2 $\mathrm{D}$ rectangular coordinate system used in rotation normalization, (c) extracted $128 \times 128$ pixel ROI, (d) estimated background image, (e) backgroundsubtracted image and, (f) after local histogram equalization performance of the system.

The recognition results summarized in Table 1 show that the method achieves better results than many conventional methods based on DCT and DFT, with a recognition accuracy of $99.3 \%$ when a $16 \times 16$ block size is used with only 16 or $6.25 \%$ of the coefficients selected per block, using a $64 \times 64$ pixel input image.

The verification results using a training set that contains 1 sample per palm from the first session and 4 samples per palm from the second session in the test set for 173 palms are shown in Table 2.

The proposed method achieves optimal verification and recognition performance when the input image is $64 \times 64$ pixels and a $16 \times 16$ block size is used for DCT feature extraction, with an Equal Error Rate (EER) of $1.11 \%$. Reduction of the input image size does not adversely affect the verification accuracy, making the proposed method well suited to real-time applications where such small image sizes would guarantee fast response times.

The proposed method offers a viable alternative to conventional methods like 'Eigenpalms' which are computationally expensive and data-dependent.

Table 1. Recognition rate (\%) for 2 block sizes as the size of the input image is reduced

\begin{tabular}{c|ccc}
\hline \hline & \multicolumn{3}{|c}{ Input image size (pixels) } \\
Block size & $128 \times 128$ & $64 \times 64$ & $32 \times 32$ \\
\hline $16 \times 16$ & 97.9 & $\mathbf{9 9 . 3}$ & 98.6 \\
$8 \times 8$ & 96.4 & 99.0 & 98.4 \\
\hline
\end{tabular}

Table 2. Equal Error Rate (\%) for two block sizes at various input image sizes

\begin{tabular}{c|ccc}
\hline \hline & \multicolumn{3}{|c}{ Input image size (pixels) } \\
Block size & $128 \times 128$ & $64 \times 64$ & $32 \times 32$ \\
\hline $16 \times 16$ & 3.33 & $\mathbf{1 . 1 1}$ & 1.36 \\
$8 \times 8$ & 2.94 & 1.14 & 1.31 \\
\hline
\end{tabular}




\title{
A Local DCT-II Feature Extraction Approach for Personal Identification Based on Palmprint
}

\author{
H. Kipsang Choge* Non-member \\ Tadahiro Oyama** Non-member \\ Stephen Karungaru* Member \\ Satoru Tsuge ${ }^{* * *} \quad$ Member \\ Minoru Fukumi* Member
}

\begin{abstract}
Biometric applications based on the palmprint have recently attracted increased attention from various researchers. In this paper, a method is presented that differs from the commonly used global statistical and structural techniques by extracting and using local features instead. The middle palm area is extracted after preprocessing for rotation, position and illumination normalization. The segmented region of interest is then divided into blocks of either $8 \times 8$ or $16 \times 16$ pixels in size. The type-II Discrete Cosine Transform (DCT) is applied to transform the blocks into DCT space. A subset of coefficients that encode the low to medium frequency components is selected using the JPEG-style zigzag scanning method. Features from each block are subsequently concatenated into a compact feature vector and used in palmprint verification experiments with palmprints from the PolyU Palmprint Database. Results indicate that this approach achieves better results than many conventional transform-based methods, with an excellent recognition accuracy above $99 \%$ and an Equal Error Rate (EER) of less than $1.2 \%$ in palmprint verification.
\end{abstract}

Keywords: palmprint recognition, local DCT, biometric feature extraction, hand-based biometrics

\section{Introduction}

The use of biometric data to establish or verify personal identity has nearly become ubiquitous in the modern world, with such diverse applications as forensics, access systems, identity cards and information security. This is because biometric identification systems are harder to circumvent and easier to use than traditional token and knowledge based systems ${ }^{(1)(2)}$.

The palmprint offers an attractive option to other metrics such as fingerprint or iris for various reasons: The print patterns on the palm are not duplicated in other people, even in the case of identical twins, and they exhibit a remarkable stability over the entire lifetime of an individual ${ }^{(3)}{ }^{(4)}$. The palm also has a larger surface area that contains an abundance of features including principal lines, wrinkles, creases and minutiae points. Because of this, lower-resolution imaging devices suffice for capturing palmprint images, thus making it more affordable than iris-based applications ${ }^{(4)-(6)}$. Unlike facebased applications where the variations in a single face can be very large due to pose, lighting, emotional and health state, palmprints from the same person exhibit

\footnotetext{
* The University of Tokushima

2-1, Minami Josanjima, Tokushima 770-8506

** Kobe City College of Technology

8-3, Gakuen-Higashimachi, Nishi-ku, Kobe Hyogo 651-2194

*** Daido University

10-3, Takiharu-cho, Minami-ku, Nagoya, Aichi 457-8530
}

a high degree of similarity. However, the variations between different palms can be very small when only the main or principal lines on the palm are considered. The challenge in palmprint recognition is therefore to effectively combine the geometrical and spatial features of the main and minor lines with local and global texture to produce an accurate representation of the palmprint in feature space.

This paper presents a holistic method that makes use of the Discrete Cosine Transform (DCT) in a novel way by exploiting its data-compacting strengths while eliminating or minimizing the weak points through careful preprocessing of the input palmprints.

This section begins with a survey of related palmprint recognition methods in the literature. The proposed method is then presented along with the motivations for choosing it and the main contribution of this work. Section 2 provides an overview of the proposed method together with the preprocessing steps, while section 3 describes the DCT feature extraction method in detail. Section 4 describes the experiments, whose results are presented and analyzed in section 5, and also compared with conventional methods. We then end the paper with some concluding remarks in section 6 .

1.1 Related Work Palmprint recognition techniques can be classified according to the type of features extracted, and these generally fall into two categories: structural and statistical methods ${ }^{(4)}{ }^{(7)}$. Statistical or holistic methods either transform the palm- 
print into a new space or consider it as a point in a multidimensional space. They include Eigenpalms and Eigenfinger $^{(2)(8)(9)}$, Fisherpalms ${ }^{(10)}$, those based on local and global texture ${ }^{(6)(11)}$, using Gabor filters ${ }^{(12)}$, and Fourier Transform ${ }^{(2)(13)(14)}$. On the other hand, structural methods extract information from structural features of palmprints such as principal lines, wrinkles, creases and minutiae and include the use of the Radon transform to extract principal lines ${ }^{(15)}$, extraction of features based on palm creases ${ }^{(16)(17)}$, extraction of structural features using wavelets and pseudo Zernike moments ${ }^{(18)(19)}$, and the use of hand geometry features ${ }^{(20)}$.

In ref. (8), Eigenpalms is used for palmprint recognition by producing a projection of the input images into "eigenspace" via the Karhunen-Loeve Transform (KLT), and using only a portion of the coefficients during matching, achieves a good recognition accuracy of $98.7 \%$ for a database of palmprints with 30 classes. Zhang ${ }^{(2)}$ used the same method on a database with 382 classes to get a recognition accuracy of $99.1 \%$ and impressive verification results.

In Fisherpalms ${ }^{(10)}$, Fisher's linear discriminant analysis is applied on a set of palmprints to find the optimal linear transformation that maximizes the Fisher criterion after first applying the KLT transform on the dataset to ensure that the within-class matrix is not singular. Although Fisherpalms and Eigenpalms perform equally well, the enormous amount of matrix manipulations necessary for obtaining the projections into KLT space and calculating the Fisher criterion make them unattractive for practical real-time applications. The data-dependence of the KLT transform also implies that a new set of coefficients need to be calculated every time new data is introduced.

Texture features have also been used successfully for palmprint feature extraction and authentication. A 2D Gabor filter adjusted to zero-DC is convolved with the input image to produce a feature vector with real and imaginary portions. Despite using low resolution palm images, the algorithm proves quite effective, with translation and rotation invariance ${ }^{(6)(12)}$.

Methods based on the Fourier Transform for palmprint recognition use either the magnitude or phase of the coefficients as feature vectors or adopt a fusion scheme that combines both. In ref. (13), the DFT image is divided into ring-like portions centered at the middle or zero frequency point to extract features representing frequency, and into slices cutting through the middle for features representing phase or direction. The two feature sets are then used in a layered manner with the radial or frequency set used at the coarse level and direction features for finer matching to achieve a recognition rate of about $95 \%$ on images from the 'PolyU' Palmprint Database ${ }^{(21)}$. Ito et al. ${ }^{(14)}$ use DFT images with a band-limited phase-only correlation function (BLPOC) and a Hamming window to compare and discriminate two palmprint images and also using images from the PolyU palmprint database, excellent verification results are presented. The major drawback of these methods is their failure to effectively account for the effects of noise because they use the whole DFT image and give equal weight to all frequency bands. This ignores the noise which is encoded in the high frequency components and can adversely affect discrimination ${ }^{(19)(22)}$. In previous work ${ }^{(23)}$, DFT features were used in combination with a genetic algorithm to select an optimal set of coefficients which are weighted in such a way that the high-frequency components are less likely to be selected. Using the same database (PolyU) a recognition accuracy above 98\% was achieved, further reinforcing the idea that DFT-related techniques can be effectively applied in palmprint recognition.

Jing and Zhang ${ }^{(24)}$ use an approach that combines DCT, KLT and Fisher's linear discriminant analysis to perform both face and palmprint recognition. The DCT is performed on preprocessed $64 \times 64$ pixel central palm area and the DCT image is then split into frequency "bands", which increase in a radial manner starting at the top left corner. Every band begins on the top edge of the image and ends on the left edge after forming a reverse L shape on the DCT image. Only the first 20 (the low frequency region) bands are selected for the next stage, which involves forming an $X \times Y$ matrix where $Y$ is the class size and $X$ is the length of the particular DCT band in consideration. All the frequency bands are ranked based on their linear separability, $J$, which is obtained by

$$
J\left(Y_{k}\right)=\frac{\operatorname{tr}\left(S_{b}\right)}{\operatorname{tr}\left(S_{w}\right)} \ldots \ldots \ldots \ldots \ldots \ldots \ldots \ldots \ldots
$$

where $Y_{k}$ is the $k^{t h}$ training feature matrix, $S_{b}$ and $S_{w}$ are the between class and within class variances respectively, and $\operatorname{tr}(\bullet)$ is the trace of the respective matrix. A threshold for $J$ is then used to select only the bands with highest separability for the next stage, which involves calculating the optimal discriminant transform $W_{\text {opt }}$ using

$$
W_{o p t}=W_{p c a} W_{f l d} \ldots \ldots \ldots \ldots \ldots \ldots \ldots \ldots
$$

where $W_{p c a}$ is calculated by selecting the principal components of $S_{t}$ which is the sum of $S_{w}$ and $S_{b}$ in Eq. 1 . On the other hand, $W_{f l d}$ is obtained using the same criterion used by the authors in Fisherpalms ${ }^{(10)}$. Finally, the Euclidean distance is used in the matching stage. This approach is basically the same as performing Fisherpalms ${ }^{(10)}$ using just the chosen frequency bands as input instead of the individual palms. Given the amount of computation needed to obtain the principal components during PCA and FLDA, and finding the within and between-class scatter, the approach is difficult to justify.

Yih et al. ${ }^{(25)}$ take a slightly different approach by first normalizing the palmprint image to $256 \times 256$ pixels and performing the DCT on $16 \times 16$ blocks. The DCT image for each block is then split into $4 \times 4$ regions, each of whose coefficients are squared and summed to obtain the DCT energy. The energy feature is arranged to form the feature vector, and using a database containing 1000 palmprint images from 100 palms, a verification accuracy of $95.4 \%$ is achieved. This method also fails to 
consider the effect of including the high-frequency components when obtaining the DCT energy of each subregion within the $16 \times 16$ block. The method is also rather inefficient as it uses all the coefficients for calculation of the DCT energy, which is equivalent to using all the pixels in the $256 \times 256$ image because the DCT is equal in size to the original image.

1.2 Proposed Method and Motivation The DCT has a close relationship to both the DFT and the KLT. The DCT very closely approaches the KLT, which is known to be the optimal transform in an information packing sense, because it minimizes the mean square error $^{(22)(26)}$. However, unlike the KLT which is datadependent and has to be redefined every time the input signal statistics change, the DCT uses preset basis functions that can be calculated beforehand, making the DCT the transform of choice in compression applications such as the JPEG standard ${ }^{(27)}$. It can also be calculated fast and efficiently using the same algorithms developed for the DFT such as the Fast Fourier Transform (FFT) by ignoring the imaginary part. Moreover, the DCT has the unique hardware advantage of having been implemented in a single integrated circuit unlike other dataindependent transforms such as the $\mathrm{DFT}^{(22)}$.

This paper seeks to exploit these advantages by using the DCT as a means of palmprint feature extraction and comparing the results with those obtained using conventional transform-based approaches. The square $128 \times 128$ region covering the central palm area is extracted from the original palmprint image and split into fixed-size sub-images on which the DCT is applied. The coefficients representing the low to medium frequencies are then selected from each block, which are then concatenated to form the feature vector that is subsequently used for palmprint classification. The coefficients are selected in the same zigzag manner used for JPEG image compression ${ }^{(27)}$, beginning from the fundamental or zero-frequency component. Because the DCT is not rotation-invariant, careful preprocessing is carried out to remove illumination and orientation variations and improve performance. The main novelty and contribution of this work is not only the successful demonstration of the application of local DCT features to palmprint recognition, but also demonstrating experimentally that the choice of input image size as well as the block-size are of paramount importance as far as recognition and verification accuracy are concerned.

Experiments are carried out to show the effectiveness of the proposed approach compared with previous methods that used DFT and DCT feature extraction for palmprint recognition. The proposed system is described in greater detail in the next two sections, where the system architecture, preprocessing and feature extraction steps are presented.

\section{Overview of System Architecture}

An overview of the proposed system is shown in Fig. 1. The enrollment and testing inputs consist of gray scale bitmap images of the palm that are not normalized for position, brightness or orientation. Before extracting the

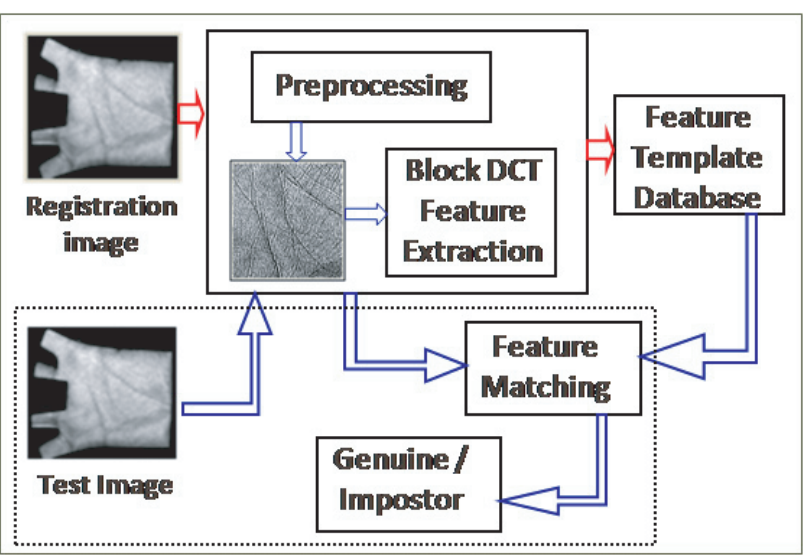

Fig. 1. Architecture for the block-based DCT palmprint feature extraction system

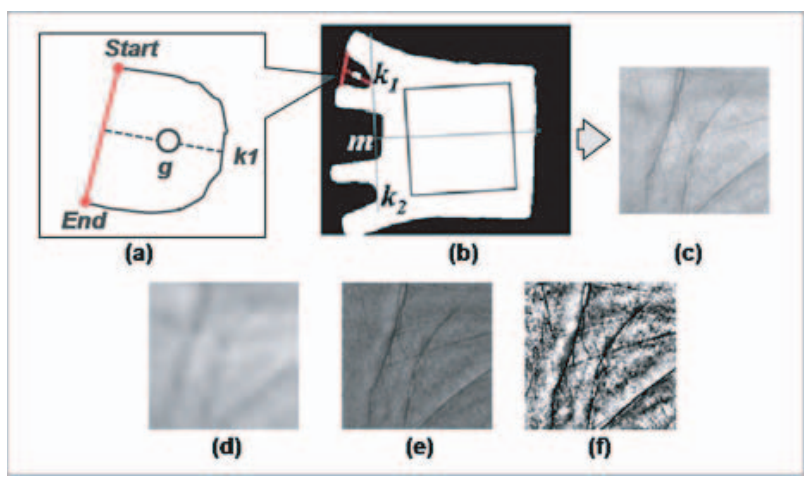

Fig. 2. The preprocessing steps used for extraction and normalization of the ROI: (a) starting and end points for calculating center of gravity, $g$, (b) the $2-\mathrm{D}$ rectangular coordinate system used in rotation normalization, (c) extracted $128 \times 128$ pixel ROI, (d) estimated background image, (e) backgroundsubtracted image and, (f) after local histogram equalization

square central palm area, it is necessary first to obtain a reference coordinate system to ensure that approximately the same area is extracted from each image. This is done in the preprocessing stage, after which a $128 \times 128$ region of interest (ROI) is segmented and used for DCT feature extraction. In testing mode, an unknown palmprint image ROI is matched to the saved templates in the database and a match found based on the minimum Euclidean distance.

2.1 Preprocessing To extract the ROI, Zhang's $\operatorname{method}^{(2)}$ is used to normalize the input image for position and rotation. The two points $k_{1}$ and $k_{2}$ shown in Fig. 2(b) are obtained on the image by first selecting a starting and ending point of the finger edge boundary between the fore and middle finger for $k_{1}$ and between the ring and little finger for $k_{2}$. An enlargement of this semi-circular background area is shown in Fig. 2(a) along with the finger edge boundary between the starting and ending points. The center of gravity of the semicircular finger edge boundary, $g$, shown in Fig. 2(a) is then calculated. Finally, a line is drawn which begins at the mid-point between the start and end points chosen earlier. The line passes through $g$ and extends to a point 
on the palmprint boundary, which is then marked as $k_{1}$ or $k_{2}$. The line joining $k_{1}$ and $k_{2}$ is used as the reference $x$ axis as shown in Fig. 2(b). A perpendicular line originating from the midpoint, $m$, of the $x$ axis is then used as the $y$ axis, and the middle square region is obtained. This $128 \times 128$ pixel region starts at a distance from point $m$ which was determined experimentally, and is used as the input palmprint for feature extraction. Because the points $k_{1}$ and $k_{2}$ are determined based on the center of the gravity of the gap between the corresponding fingers, this region is approximately the same in each palmprint image.

2.1.1 Brightness Normalization This is necessary because the brightness was allowed to vary both during and between the two sessions of capturing the palmprint images in the database. A coarse estimate of the background illumination is first obtained by finding the average of every $8 \times 8$ region over the entire ROI ${ }^{(28)}$. Each average value forms a pixel in a $16 \times 16$ image (because the ROI size is $128 \times 128$ pixels), which is then expanded to the same size as the ROI using bi-cubic interpolation to obtain the estimated background image shown in Fig. 2(d). The estimated background is then subtracted from the original image to produce a brightness-normalized image, shown in Fig. 2(e). Finally, contrast improvement is achieved by local histogram equalization using $16 \times 16$ blocks as shown in Fig. 2(f), which though visually less pleasing, has a more evenly distributed histogram than the brightnessnormalized image.

\section{Block-based DCT Feature Extraction}

Feature vectors that represent the palms in the database are obtained by computing the DCT of the preprocessed images. The $2-\mathrm{D}$ type-II DCT, $C(u, v)$, of an image $I(r, c)$ which is $N \times N$ pixels in size, is twice the length of the DFT and is given by

$$
\begin{aligned}
C(u, v)= & \alpha_{u} \alpha_{v} \sum_{r=0}^{N-1} \sum_{c=0}^{N-1} I(r, c) \cos \left[\frac{(2 r+1) u \pi}{2 N}\right] \\
& \cos \left[\frac{(2 c+1) v \pi}{2 N}\right] \ldots \ldots \ldots \ldots \ldots \ldots
\end{aligned}
$$

where $u$ and $v$ are the coordinates in the DCT domain while $r$ and $c$ are the spatial coordinates and

$$
\begin{aligned}
\alpha_{u}, \alpha_{v} & =\sqrt{\frac{1}{N}} \text { for } u, v=0 \text { and } \\
& =\sqrt{\frac{2}{N}} \text { for } u, v=1,2, \ldots N-1 \ldots \ldots
\end{aligned}
$$

Two samples of extracted ROIs from the palmprint database are shown in Fig. 3(a). After resizing the images to $64 \times 64$ pixels, the DCT is performed on $16 \times 16$ blocks and only the first 16 coefficients scanned diagonally from the top left corner are retained and plotted in Fig. 3(b). The first sample is plotted in dotted blue color while the second one is plotted in solid red color. It is seen from the graphs that the DCT coefficients for different palms are different in both magnitude direction.

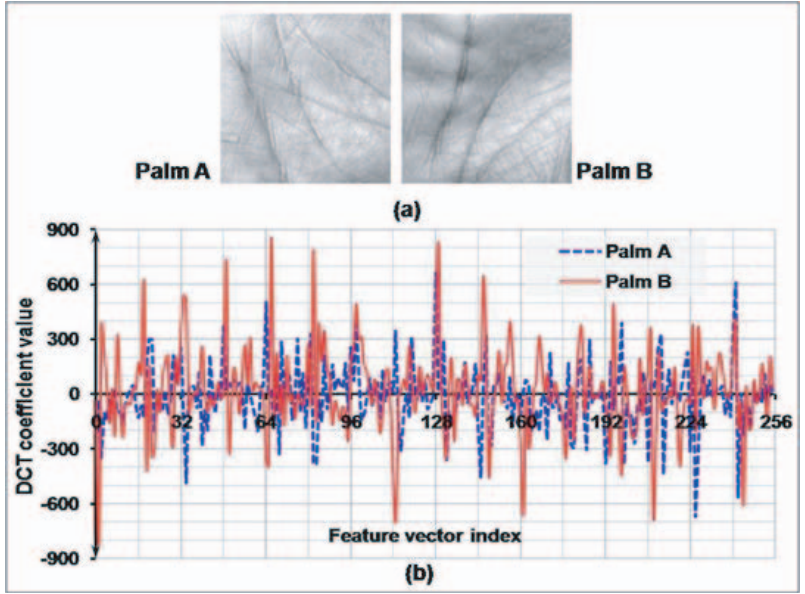

Fig. 3. DCT features using $16 \times 16$ blocks for different palms: (a) palmprint samples of two individuals and, (b) plot of the first 16 DCT coefficients from each block for both palms

3.1 DCT Block Size In the proposed method, the input image is split into fixed-size blocks which are either $16 \times 16$ or $8 \times 8$ pixels in size prior to performing DCT on each of the blocks. The aim for doing this is twofold: to determine the optimal block size for a fixed input image size and to investigate the relationship between the block size and the input image size as far as palmprint verification performance is concerned. The JPEG standard ${ }^{(27)}$ uses $8 \times 8$ blocks for DCT, and optimized fast algorithms have been developed specifically for this size, but we would like to compare the results from using a larger block size as well.

3.2 Palmprint Input Image Size The original $128 \times 128$ pixel ROI is initially used for DCT feature extraction using the two block sizes defined above. Next, the size is gradually reduced via bi-cubic interpolation down to $64 \times 64$ and finally to $32 \times 32$ pixels. For each image size, feature extraction and matching is performed and the results are compared to those obtained when using the original ROI. The effect of gradual size reduction on verification accuracy has not been investigated rigorously enough in other transform-based methods in the literature, despite the enormous improvement in speed of processing that could be achieved in this way. A $50 \%$ reduction in input image size would increase the speed of computation of the system by a factor of 4 . The results obtained from these experiments can therefore be evaluated based on optimizing not only the block size, but also the input image size and hence the speed of processing.

\subsection{Zigzag Scanning of DCT Coefficients}

The DCT is known to represent most natural images efficiently because it concentrates most of the significant coefficients on or near the top left corner of the DCT domain image. The coefficients are uncorrelated and represent increasing frequencies in a diagonal manner starting with the fundamental or zero-frequency value at the top left corner, which from Eq. 3 represents the average brightness value over a given $N \times N$ block.

In our experiments, we adopt the zigzag scan similar 


$\left[\begin{array}{ccccccccc}1109 & 468 & 361 & -294 & -26 & 65 & 30 & -36 \\ 17 & 226 & -248 & -99 & 4 & 68 & -46 & -44 \\ -124 & -53 & -8 & 85 & 12 & -53 & 46 & -24 \\ -33 & 59 & -14 & -37 & -55 & -35 & 24 & 8 \\ -16 & -66 & -8 & 26 & -25 & 50 & -21 & -5 \\ 2 & -17 & 32 & -21 & -13 & -30 & -8 & 19 \\ 5 & -17 & -4 & 13 & -11 & 8 & -25 & 20 & 14 \\ 19 & 15 & -10 & -5 & 24 & -41 & 24 & -14\end{array}\right]$

Fig. 4. The $2-\mathrm{D}$ DCT of an $8 \times 8$ block. The dashed line shows the zigzag scanning method used

to that used in image compression, because it guarantees that coefficients belonging to the same frequency bands will be close or next to each other, and in this way we can effectively exclude the high frequency components which are highly susceptible to noise. In previous experiments ${ }^{(29)}$, a similar method to that used by Hafed et al. ${ }^{(26)}$ was applied whereby the DCT coefficients were ranked based on magnitude and the largest ones selected. In Fig. 4 the $2-\mathrm{D}$ DCT of an $8 \times 8$ block is shown together with the zigzag scanning style used to select the top $50 \%$ coefficients. It can be observed that if the coefficients are ranked based on magnitude, those highlighted by small boxes in the figure would be selected and included in the feature vector, even though they belong in the noise-prone high-frequency area of the sub image.

3.4 Feature Matching In the matching stage, a nearest-neighbor classifier is used based on minimizing the total Euclidean distance, $D$, which corresponds to the summation of the Euclidean distance, $d$, between the feature vector from each block of the unknown palm, $b$, and that from the corresponding block in the database image, $a$.

$$
D=\sum_{j=1}^{M} d_{j} \ldots \ldots \ldots \ldots \ldots \ldots \ldots \ldots \ldots \ldots \ldots
$$

where $M$ is the number of blocks per image and $d$ is given by

$$
d=\sqrt{\sum_{i=1}^{L}\left(a_{i}-b_{i}\right)^{2}} \cdots \ldots \ldots \ldots \ldots \ldots \ldots \ldots
$$

where $L$ is the length of the feature vector per block. This corresponds to the number of coefficients retained after zigzag scanning.

\section{Experiments}

4.1 Palmprint Database Palmprint images from the Hong Kong Polytechnic University palmprint database ('PolyU Palmprint Database') (21) are used in experiments to evaluate the proposed method. The database contains between 7 and 10 samples of the right and left palm per individual in bitmap format with an original size of $384 \times 284$ pixels. The images are scanned at a resolution of 72 dots per inch in two sessions approximately 2 months apart, with an approximately equal number of images captured at each session. Guiding pegs are used for positioning the fingers but they do not completely eliminate variations in orientation. The illumination is also allowed to vary slightly between different palmprint capture sessions.

4.2 Experimental Conditions Two experimental formats are used in order to test the performance of the proposed method in a recognition or one-to-many matching mode and also one-to-one or palmprint authentication mode. For both formats, palmprints belonging to 173 individuals are used to create the training and testing sets.

The preprocessing method, as described in Sect. 2, uses two reference points to obtain a new coordinate axis for position normalization. However, on some of the palmprints in the database, the background area between the fingers of interest could not be properly segmented and in some cases the area does not exist at all because the individual failed to spread their fingers during the capture process. Such samples are considered invalid and excluded from the dataset.

4.2.1 Palmprint Recognition For palmprint recognition experiments, a training or template set is created by selecting and preprocessing 1 sample from the first session per palm for the 173 individuals. A testing or evaluation set is then formed that consists of 4 samples per palm from the second session for the same set of individuals so that the test set contains a total of $173 \times 4=692$ samples. This reflects a real world biometric system in which the saved template is usually captured at an earlier date than the sample that is presented to the system for identification. A total of 173 matching attempts are made for each test sample to give a total of 119,716 matching attempts.

4.2.2 Palmprint Authentication This is the mode in which a majority of biometric systems operate, such as access or security applications where a sample is presented to the system together with a claimed identity. The system is then required to authenticate or verify that the sample indeed belongs to the individual (genuine) or does not (impostor) by matching the sample to a saved template based on some known similarity measure.

The same training and test set used in recognition is used to evaluate the verification accuracy of the system, so that the session attribute is maintained for both experimental formats. However, during verification mode, each of the 692 samples in the test set (which are from the second session) is matched to all the 173 samples in the template set at a given threshold. A total of 119,716 matching attempts are made per threshold, of which $173 \times 4=692$ are genuine matches while the rest 119,024 are impostor matches. The threshold is then increased and the whole process repeated again.

4.2.3 Performance Evaluation Criteria The recognition accuracy of the system is evaluated based on the number of test samples in the recognition set which are correctly identified, i.e. those whose minimum distance is achieved with a template palmprint belonging to the same individual. 
During verification, False Rejection Rate (FRR) measures the rate at which genuine samples are rejected as impostors. This occurs if the Euclidean distance between the unknown sample and the claimed template sample is greater than the threshold value. The False Acceptance Rate (FAR) is the rate at which impostors are accepted as genuine. The FRR and FAR are given by

$$
\begin{aligned}
& F R R(\%)=\left(\frac{R G}{T G A}\right) \times 100 \ldots \ldots \ldots \ldots \ldots \ldots \\
& F A R(\%)=\left(\frac{A F}{T F A}\right) \times 100 \ldots \ldots \ldots \ldots \ldots
\end{aligned}
$$

where RG denotes the number of genuine palmprints rejected as fake, AF represents the number of impostors accepted as genuine while TGA and TFA denote the total genuine attempts and total fake matching attempts respectively.

Receiver Operating Characteristic (ROC) curves, which plot the FRR against the FAR are used to obtain the Equal Error Rate (EER). This indicates the optimal operating point where both FRR and FAR are minimum. The Verification Accuracy (VA) ${ }^{(25)}$ gives an indication of the overall verification performance of the system and is obtained from the FRR and FAR as

$$
V A(\%)=100-\left(\frac{F A R+F R R}{2}\right) \cdots \cdots \cdots
$$

\section{Results and Analyses}

The recognition accuracy of the proposed method using two block sizes for DCT feature extraction and 3 input image sizes is shown in Fig. 5. The number of coefficients retained after the zigzag scan and concatenated to form the feature vector is varied downwards from $50 \%$ and the results shown in Fig. 5 give the maximum values for recognition accuracy. The highest recognition accuracy of $99.3 \%$, where a total of 687 out of the 692 samples in the test set are correctly identified, is achieved with an input image size of $64 \times 64$ pixels and a $16 \times 16$ block size during DCT feature extraction. The number of coefficients retained after zigzag scanning in this case is $6.25 \%$ or just 16 per block, including the zero-frequency component. It is interesting to note that using the original input image size of $128 \times 128$ pixels produces a lower recognition rate of $97.9 \%$, while the smallest input image size of $32 \times 32$ pixels achieves more than $98 \%$ accuracy.

Table 1 shows the variation in the recognition accuracy for a $64 \times 64$ input image as the number of coefficients retained in each block is reduced from $50 \%$ to $3.125 \%$ for both blocks. It is noted that as the size of the subset of DCT coefficients retained for use in the feature vector is reduced, the performance of the systems gradually improves up to a certain point, after which it starts to decay. This phenomenon implies that although the data compression ratio has reduced beyond that which is required for an accurate reconstruction of the original palmprint, the performance of the system has not been affected adversely. A similar observation

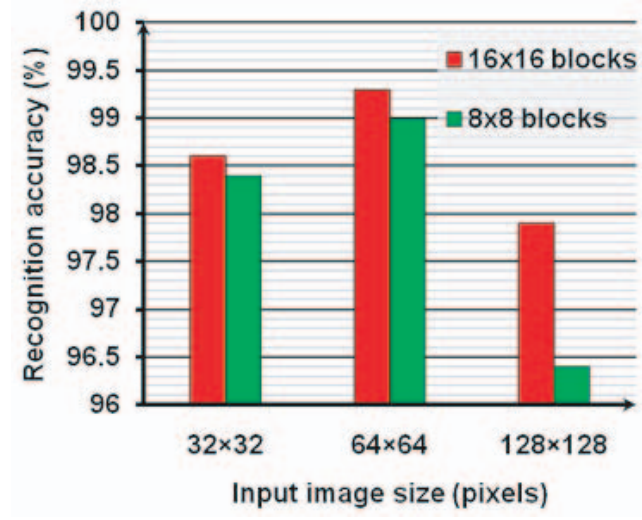

Fig. 5. Recognition accuracy (\%) for $8 \times 8$ (green), and $16 \times 16$ (red) blocks over 3 input image sizes

Table 1. Recognition rate (\%) for 2 block sizes when reducing the number of retained coefficients

\begin{tabular}{c|cc}
\hline \hline \multirow{2}{*}{ Retained coefficients per block (\%) } & \multicolumn{2}{|c}{ Recognition rate (\%) } \\
& $16 \times 16$ blocks & $8 \times 8$ blocks \\
\hline 50 & 96.7 & 97.0 \\
25 & 97.3 & 97.8 \\
12.5 & 98.4 & 98.6 \\
6.25 & 99.3 & 99.0 \\
3.13 & 98.7 & 98.7 \\
\hline
\end{tabular}

Table 2. EER (\%) of the proposed method for 2 blocks and 3 input image sizes

\begin{tabular}{c|ccc}
\hline \hline \multirow{2}{*}{ Block size } & \multicolumn{3}{|c}{ EER (\%) } \\
& $128 \times 128$ & $64 \times 64$ & $32 \times 32$ \\
\hline $16 \times 16$ & 3.33 & $\mathbf{1 . 1 1}$ & 1.36 \\
$8 \times 8$ & 2.94 & 1.14 & 1.31 \\
\hline
\end{tabular}

Table 3. Verification accuracy (\%) for 2 blocks and 3 input image sizes

\begin{tabular}{c|ccc}
\hline \hline \multirow{2}{*}{ Block size } & \multicolumn{3}{|c}{ Verification accuracy } \\
& $128 \times 128$ & $64 \times 64$ & $32 \times 32$ \\
\hline $16 \times 16$ & 96.9 & $\mathbf{9 9 . 1}$ & 98.6 \\
$8 \times 8$ & 97.1 & $\mathbf{9 9 . 1}$ & 98.7 \\
\hline
\end{tabular}

was made in previous research using DCT for face recognition ${ }^{(26)}$ and KLT features for image retrieval ${ }^{(30)}$ where accurate reconstruction was found not be a necessary requirement for good recognition performance, and an increase in the number of retained coefficients failed to improve the accuracy.

The proposed method therefore produces optimal palmprint recognition accuracy above $99 \%$ when an input image size of $64 \times 64$ pixels is used together with a $16 \times 16$ block size for DCT feature extraction, although an $8 \times 8$ block works just as well. In either case, $6.25 \%$ of the coefficients are retained per block, for a compact feature vector whose length is 256 .

5.1 Palmprint Verification Results The error rates of the proposed system are shown by the ROC curves plotted in Fig. 6 , where only the $16 \times 16$ block size is shown for clarity. The EER for both blocks at the three input image sizes is summarized in Table 2, while the verification accuracy calculated using Eq. 9 is shown in Table 3.

From Table 2, it is observed that both block sizes pro- 


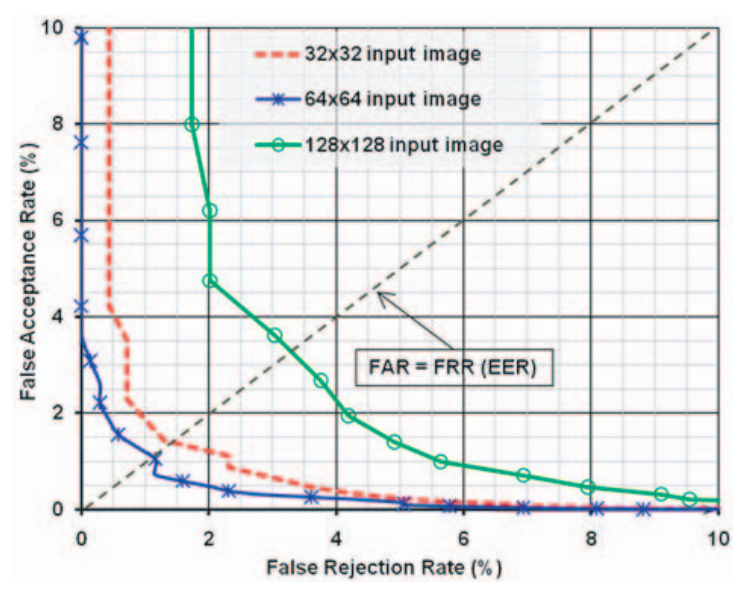

Fig. 6. ROC curves for a $16 \times 16$ pixel block and 3 input image sizes. The dotted diagonal line shows the EER

duce the lowest EER when the input image size is $64 \times 64$ pixels, at $1.11 \%$ and $1.14 \%$ for the $16 \times 16$ and $8 \times 8$ block sizes respectively. This value decays only slightly when the input image is further reduced to $32 \times 32$ pixels, but a bigger deterioration is observed at the larger $128 \times 128$ pixel image size, with the EER increasing to $3.33 \%$ for the $16 \times 16$ block size.

The maximum verification accuracy of the proposed method, shown in Table 3, is $99.1 \%$, and is achieved for both blocks at $64 \times 64$ input image size. Whereas the EER indicates the point in the ROC curves where the FAR is equal to the FRR, the verification accuracy indicates the point at which the system is achieving the maximum success in verification and is not necessarily when FAR and FRR are equal. The $99.1 \%$ value shown in Table 3 for the $16 \times 16$ block, for example, is achieved when the $\mathrm{FAR}=0.72 \%$ and $\mathrm{FRR}=1.16 \%$. For a highsecurity system where the cost of false acceptances is higher, this would represent a better operating point than the EER point ${ }^{(2)}$.

It should be noted that similarly to recognition, the optimal values for verification are obtained when fewer coefficients are retained after zigzag scan, rather than more. The values shown in Tables 2 and 3 are achieved when $6.25 \%$ of the coefficients are retained. However, when $12.5 \%$ and $25 \%$ of the coefficients are retained, no change in the EER is observed, but if increased to $50 \%$, the EER starts to increase, just as it does when less than $6.25 \%$ of the coefficients are retained.

5.2 Performance Comparison with Conventional Methods The DCT is closely related to the DFT, and a few methods exist in the literature where both transforms have been used, alone or in combination with other techniques, for palmprint recognition with varying results ${ }^{(13)(23)-(25)}$. To test the effectiveness of the proposed method, it is necessary to compare it against closely related conventional methods, briefly summarized here for clarity.

5.2.1 Comparison with DFT and Yih's Approach To compare the proposed method with the DFT, the same dataset is used but the block-DCT is re-
Table 4. Comparison of recognition accuracy (\%) for 3 approaches using 3 input image sizes

\begin{tabular}{|c|c|c|c|}
\hline Approach & \multicolumn{3}{|c|}{$\overline{\text { Recognition accuracy (\%) for input image: }}$} \\
\hline Proposed-I & 96.4 & 99.0 & 98.4 \\
\hline Proposed-II & 97.8 & 99.3 & 98.6 \\
\hline DFT-I & 97.1 & 99.0 & 96.1 \\
\hline DFT-II & 99.0 & 98.7 & 94.1 \\
\hline Yih-I & 92.6 & 91.2 & 65.2 \\
\hline Yih-II & 86.3 & 85.8 & 59.1 \\
\hline
\end{tabular}

placed with block-DFT for feature extraction. However, because the DFT is roughly half the length of the DCT, all the magnitudes of the DFT coefficients are used in place of the $50 \%$ used in the proposed method to allow for fair comparison.

Yih et al. ${ }^{(25)}$ performed a comparative study of three approaches for palmprint recognition-DCT energy, Wavelet Transform and an approach using the Sobel edge detector, dubbed "SobelCode". The input images used in their study were captured by digital camera in RGB format and an original size of $1024 \times 768$ pixels. After orientation normalization, a $256 \times 256$ ROI was extracted from the central palm area and used as input to each of the three methods.

To extract the DCT energy features, they divided the ROI into $16 \times 16$ blocks, applied the DCT on each block and then split the $16 \times 16$ DCT coefficient matrix further into $4 \times 4$ regions so that each DCT block is divided into 16 regions. For each $4 \times 4$ region, the DCT energy is calculated as the squared sum of all the coefficients in the region. By doing this in every DCT block, they end up with a $64 \times 64$ matrix whose values consist of the squared sums of the DCT coefficients in each region, which is similar theoretically to resizing the original image by summing up $4 \times 4$ neighborhoods, except that this is done in the DCT domain.

For comparison to Yih's approach, the same steps are carried out using the same dataset as the proposed method. Two DCT energy feature vectors are created using $4 \times 4$ and $2 \times 2$ regions of a $16 \times 16$ DCT block. This is because the largest extracted ROI is half the size extracted by Yih et al. (their original images are more than twice the size of those in the PolyU database) and we would like to have the same feature vector size.

The results from recognition and verification experiments using DFT magnitude features, Yih's approach, and the proposed method are summarized in Tables 4 and 5 respectively. In both tables, DFT and the proposed method using $8 \times 8$ blocks are marked DFT-I and Proposed-I respectively while DFT and the proposed method using $16 \times 16$ blocks are marked DFT-II and Proposed-II respectively. Yih's approach using $2 \times 2$ and $4 \times 4$ regions are marked Yih-I and Yih-II respectively.

From Table 4, the maximum recognition accuracy of $92.6 \%$ from Yih's method is achieved with the largest input image size of $128 \times 128$ pixels and feature vector length of $32 \times 32=1024$, while the proposed method, at a feature length of 256 achieves $99.3 \%$ accuracy. On the other hand, using DFT features produces impressive 
Table 5. Verification EER (\%) of various approaches over 3 input image sizes

\begin{tabular}{|c|c|c|c|}
\hline Approach & \multicolumn{3}{|c|}{ EER (\%) for each input image size: } \\
\hline Proposed-I & 2.94 & 1.14 & 1.31 \\
\hline Proposed-II & 3.33 & 1.11 & 1.36 \\
\hline DFT-I & 1.70 & 1.20 & 2.02 \\
\hline DFT-II & 1.31 & 1.80 & 2.68 \\
\hline Yih-I & 6.87 & 7.13 & 9.8 \\
\hline Yih-II & 11.14 & 11.45 & 13.3 \\
\hline
\end{tabular}

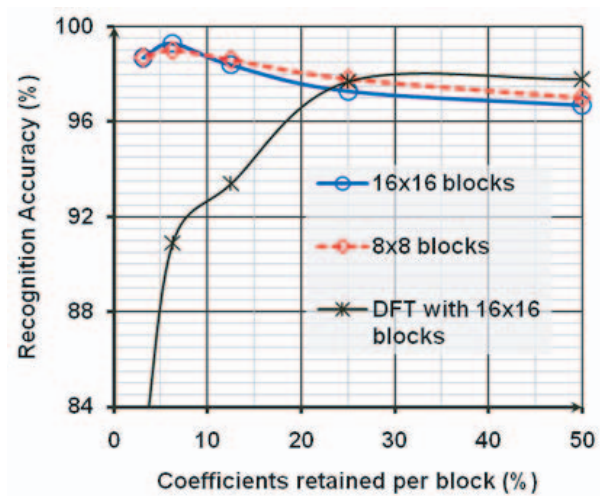

Fig. 7. A comparison of recognition accuracy between the proposed method and DFT features. " $16 \times 16$ blocks" and " $8 \times 8$ blocks" plots represent the proposed method for the respective block sizes. All the plots are obtained using a $64 \times 64$ pixel input image

$99.1 \%$ accuracy when the original ROI size is used together with a $16 \times 16$ block size and also when a $64 \times 64$ input image is used with $8 \times 8$ blocks for DFT. However, compared to the proposed method, the accuracy of DFT features decays rapidly when the input image size is reduced further to $32 \times 32$ pixels, as it does for Yih'g approach, whose accuracy falls to $55.2 \%$ at the smallest input image size. This is expected theoretically, because Yih's method essentially averages neighborhoods and is not effective when the image size is smaller and the loss of information from summing up $2 \times 2$ or $4 \times 4$ pixel blocks becomes more pronounced. In Table 5 , the optimal EER for each approach, highlighted in boldface, shows that the proposed method and DFT features achieve comparable EERs of $1.11 \%$ and $1.2 \%$ respectively at a $64 \times 64$ pixel input image size.

Figure 7 shows the variation of the recognition accuracy with the number of coefficients retained per block, and it can be seen that whereas the accuracy improves with a decrease in the retained coefficients for the proposed method, the reverse is true when using DFT features. This is because DFT is not known to concentrate most of the information in the low-frequency region as much as the DCT does. This property made the DCT a better choice for the JPEG compression scheme, where the zigzag scan is also used to ensure that low frequencies are arranged close together in the extracted onedimensional array ${ }^{(27)}$.

In order to compare the visual quality of palmprint images reconstructed after discarding some of the coefficients, DCT and DFT are carried out using $16 \times 16$

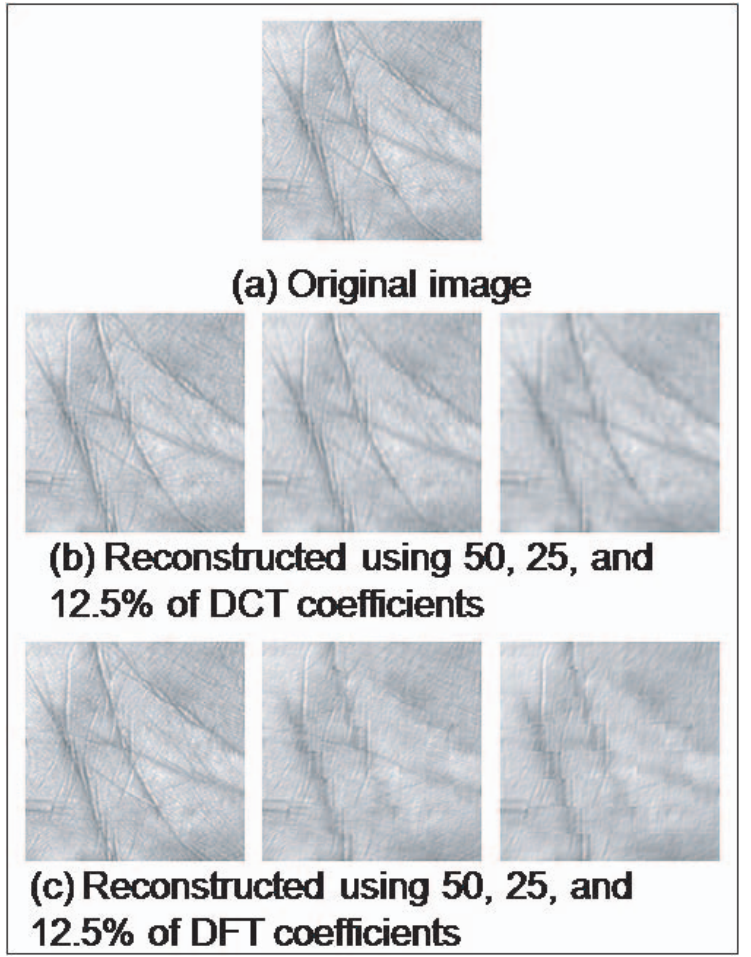

Fig. 8. Palmprint images reconstructed using a subset of the DFT and DCT coefficients

blocks, after which most of the medium and high frequency coefficients are discarded prior to reconstruction. Figure 8 shows the reconstructed images after retaining 50,25 , and $12.5 \%$ of the DCT and DFT coefficients and discarding the rest. It can be seen from Fig 8 (b) and (c) that as more and more coefficients are discarded, the visual quality remains good for the DCT while it deteriorates severely when the DFT is used.

Although the order of complexity for calculating the DFT and DCT is similar, the proposed method is more suited for real-time applications because of the smaller feature vector length of 256 compared with 2048 (32 coefficients $\times 64$ blocks) used for DFT to achieve the optimal EER. Also, DCT is calculated with only real numbers while DFT involves complex-domain calculations as well. It is interesting to note as well that reduction of the input image down to $32 \times 32$ pixels only reduces the recognition accuracy by $0.7 \%$ to $98.6 \%$ for the proposed method unlike the DFT, which experiences a $2.9 \%$ reduction in recognition accuracy over the same input image reduction. This implies that for real-time applications, a $32 \times 32$ palmprint ROI could be used for an access system without greatly compromising the security, while achieving a 16-fold improvement in speed compared to a system that would use a $128 \times 128$ input image.

5.2.2 Other Conventional Methods Jing's approach $^{(24)}$, summarized in Sect. 1.1, also uses the lower frequency region of the DCT for feature extraction, although in their case they use the global DCT. Using 5 training samples per palm from the first session and 5 from the second, they achieve an average recognition accuracy of $97.7 \%$, with a feature vector length of 
181 and a dataset of 190 different palms. No palmprint verification results are reported. The proposed method, using the same database and only one sample from the first session for training, applies the DCT in a straightforward manner to achieve a higher recognition accuracy.

In terms of computation, the order of complexity of PCA for an $X \times Y$ feature matrix is $O\left(M^{\prime} Y^{2}\right)$ where $M^{\prime}$ is the number of basis vectors and $Y$ is the class size, while that of the DCT is $O\left(N^{2} \log _{2} N\right)$, where $N$ is the image size given in Eq.3. Even when the number of blocks is taken into consideration for the proposed method, it should be noted that Jing's method not only computes the PCA but also Fisherpalms, together with associated matrix manipulations to obtain the between and within class variances, which can make the approach intractable and unsuited to real-time applications.

In other conventional methods, Wen-xin et al. ${ }^{(2)(13)}$ used global DFT features on the same database and despite using two feature sets to represent direction and frequency, the maximum recognition accuracy of $95.5 \%$ and EER of $3.6 \%$ were obtained. Global DFT features wera also used by the authors ${ }^{(23)}$, and by using a genetic algorithm (GA) to select an optimal set of coefficients, a recognition accuracy above $98 \%$ was achieved using the same palmprint database but extra overhead is incurred from applying the GA. Other methods such as Eigenpalms and Fisherpalms require the use of large matrices to compute the basis vectors during training in order to transform the training set into the new KLT space. Both methods also require the recalculation of eigenvalues and eigenvectors every time new data is introduced. This makes such methods computationally expensive.

\section{Conclusions}

A holistic method of palmprint feature extraction and matching based on block-DCT features was presented. Several experiments were conducted to test the effectiveness of the method in recognition and verification using images from the 'PolyU' Palmprint Database, clearly demonstrating that the use of local DCT features for palmprint recognition is an effective alternative to other statistical or structural methods. Experiments also showed that careful preprocessing mitigates or eliminates the effects of slight variations in position and orientation in the input images, and that the proposed method is robust to input image size.

Excellent recognition results are obtained when a $16 \times 16$ block size and a $64 \times 64$ pixel input image are used. In this case, using just 256 or $6.25 \%$ of the DCT coefficients from the low to medium frequency part of the DCT image produces a recognition accuracy of $99.3 \%$. The best EER of the proposed method is $1.11 \%$ while a maximum verification accuracy of $99.1 \%$ is achieved.

In future work, an improved preprocessing method is investigated because the method used here for position normalization relies on the ability to properly segment the background areas between the fingers. In some of the images in the database, these areas are partly occluded, which adversely affects the accuracy of normalization as approximate borders are used in such cases. The use of overlapping blocks for DCT feature extraction is investigated as well, in order to further minimize the effects of slight variations in position and orientation.

\section{Acknowledgment}

Portions of the research in this paper use the 'PolyU' palmprint database collected by Hong Kong Polytechnic University.

(Manuscript received Nov. 11, 2009, revised April 19, 2010)

\section{References}

(1) A. K. Jain and S. Pankanti: "Beyond Fingerprinting", in Scientific American, September 2008 Issue, pp.78-81 (2008)

(2) D. D. Zhang: Palmprint Authentication, Kluwer Academic Publishers, USA (2004)

( 3 ) G. K. O. Michael, T. Connie, and A. B. J. Teoh: "Touch-less Palm print Biometrics: Novel Design and Implementation", Image and Vision Computing, Vol.26, pp.1551-1560 (2008)

(4) T. Connie, A. Beng Jin, M. Ong, and D. Ling: "An Automated Palmprint Recognition System", Image and Vision Computing, Vol.23, pp.501-515 (2005)

(5) W. Shu and D. Zhang: "Automated Personal Identification by Palmprint", Optical Engineering, Vol.37, No.8, pp.2359-2362 (1998)

(6) D. Zhang, W. Kong, J. You, and M. Wong: "Online Palmprint Identification", Trans. on Patt. Analysis and Mach. Intel, Vol.25, No.9, pp.1041-1050 (2003)

( 7 ) J. Shen, Y. Moon, and H. Yeung: "Palmprint Authentication using Time Series", In: T. Kanade, A. Jain, and N. K. Ratha (eds), AVBPA 2005, LNCS 3546, pp.376-385 (2005)

(8) A. Kumar and H. C. Shen: "Recognition of Palmprint using Eigenpalms", Proc. of CVPRIP, North Carolina (2003)

(9) S. Ribaric and I. Fratric: "A Biometric Identification System Based on Eigenpalm and Eigenfinger Features", IEEE Trans. Patt. Analysis. and Mach. Intell., Vol.27, No.11, pp. 16981709 (2005)

(10) X. Wu, D. Zhang, and K. Wang: "Fisherpalms Based Palmprint Recognition", Pattern Recognition Letters 24, pp.28292838 (2003)

(11) J. You, W. Li, and D. Zhang: "Hierarchical Palmprint Identification via Multiple Feature Extraction", Pattern Recognition 35, pp.847-859 (2002)

(12) W. K. Kong, D. Zhang, and W. Li: "Palmprint Feature Extraction using 2-D Gabor Filters", Pattern Recognition 36, pp. 2339-2347 (2003)

(13) L.Wen-xin, D. Zhang, and X. Zhuo-qun: "Palmprint Recognition Based on Fourier Transform", Journal Of Software, Vol.13, No.5, pp.879-886 (2002)

(14) K. Ito, T. Aoki, H. Nakajima, K. Kobayashi, and T. Higuchi: "A Palmprint Recognition Algorithm using Phase-Only Correlation", IEICE Trans. Fundamentals, Vol.E91-A, No.4, pp.1023-1030 (2008)

(15) D. Huang, W. Jia, and D. Zhang: "Palmprint Verification Based on Principal Lines", Pattern Recognition, Vol.41, pp.1316-1328 (2008)

(16) J. Chen, C. Zhang, and G. Rong: "Palmprint Recognition using Crease", Proc. of Intl. Conf. on Image Processing, pp.234237 (2001)

(17) J. Funada, N. Ohta, M. Mizoguchi, T. Temma, K. Nakanishi, A. Murai, T. Suiguchi, T. Wakabayashi, and Y. Yamada: "Feature Extraction Method for Palmprint Considering Elimination of Creases", Proc. of 14th Intl. Conference on Pattern Recognition, Vol.2, pp.1849-1854 (1998)

(18) J. Wu, X. You, Y. Y. Tang, and W. Cheung: "Palmprint Identification Based on Non-separable Wavelet Filter Banks", Proc. of 19th IEEE ICPR, pp.1-4 (2008)

(19) Y. Pang, A. T. B. Jin, and D. N. C. Ling: "Palmprint Authentication System using Wavelet Based Pseudo Zernike Moments Features", International Journal of CIM, Vol.13, No.2, 
pp.13-26 (2005)

(20) A. Kumar, D. C. M. Wong, H. C. Shen, and A. K. Jain: "Personal Verification using Palmprint and Hand Geometry Biometric", LNCS 2688, pp.668-678 (2003)

(21) Hong Kong Polytechnic ("PolyU") Palmprint Database, http://www.comp.polyu.edu.hk/ ${ }^{\sim}$ biometrics

(22) C. Gonzalez and R. E. Woods: Digital Image Processing, 2nd Ed., Prentice Hall, NJ, USA (2002)

(23) H. K. Choge, T. Oyama, S. Karungaru, S. Tsuge, and M. Fukumi: "A DFT-Based Method of Feature Extraction for Palmprint Recognition", IEEJ Trans. Electronics, Information and Systems, Vol.129, No.7, pp.1296-1304 (2009)

(24) X. Jing and D. Zhang: "A Face and Palmprint Recognition Approach Based on Discriminant DCT Feature Extraction", IEEE Trans. Systems, Man, and Cybernetics-Part B: Cybernetics, Vol.34, No.6, pp.2405-2415 (2004)

(25) E. W. K. Yih, G. Sainarayanan, and A. Chekima: "Palmprint Based Biometric System: A Comparative Study on Discrete Cosine Transform Energy, Wavelet Transform Energy and SobelCode Methods", Intl. J. on Biomedical Soft Comp. and Human Sci., Vol.14, No.1, pp.11-19 (2009)

(26) Z. M. Hafed and M. D. Levine: "Face recognition Using the Discrete Cosine Transform", Intl. Journal of Computing, Vol.43, No.3, pp.167-188 (2001)

(27) G. K. Wallace: "The JPEG Still Picture Compression Standard", IEEE Trans. Consumer Electronics, Vol.38, No.1, pp.xvii-xxxiv (1992)

(28) L. Ma, T. Tan, Y. Wang, and D. Zhang: "Personal Identification Based on Iris Texture Analysis", IEEE Trans. PAMI, Vol.25, No.12, pp.1519-1533 (2003)

(29) H. K. Choge, T. Oyama, S. Karungaru, S. Tsuge, and M. Fukumi: "Palmprint Authentication using Localized DCT Feature Extraction", Proc. of IEEJ EIS Conference, pp.746$750(2009)$

(30) D. L. Swets and J. Weng: "Using Discriminant Eigenfeatures for Image Retrieval", IEEE Trans. PAMI, Vol.18, No.8, pp.831-836 (1996)

H. Kipsang Choge (Non-member) received the B.Sc. de-

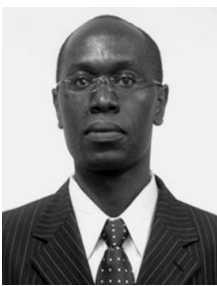
gree in Electrical and Electronic Engineering from Jomo Kenyatta University of Agriculture and Technology (JKUAT), Kenya, in 1998. In 2003, he received his MS Degree in Electrical Engineering from Southern Illinois University at Edwardsville, USA, and joined his former department at JKUAT in 2005 as an Assistant Lecturer. He is currently a Ph. D. student at the Graduate School of Advanced Technology and Science, The University of Tokushima. His research interests include signal and image processing, genetic algorithms, neural networks, pattern recognition and biometrics. He is a member of the Institute of Electrical and Electronic Engineers (IEEE) and IEEE Computer Society.
Tadahiro Oyama (Non-member) received the B.E. degree from the National Institution for Academic Degrees and University Evaluation, in 2005, and M.E. degree from The University of Tokushima in 2007 where he also received his $\mathrm{PhD}$ in System Innovation Engineering in 2010. He is currently an assistant professor at Kobe National College of Technology. His research interests include signal and image processing. He is a member of the IEICE.

Stephen Karungaru (Member) received the B.Sc. degree

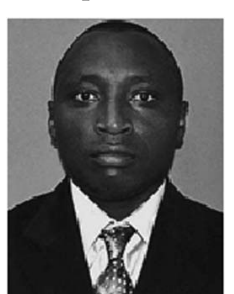
in electronics and communication from Moi University in 1992. He then joined the Department of Electrical and Electronics Engineering, Jomo Kenyatta University of Agriculture and Technology as a teaching assistant. He received the M.E. degree and a $\mathrm{PhD}$ in information system design from the Department of Information Science and Intelligent Systems, University of Tokushima in 2001 and 2004 respectively. He is currently an Assistant Professor in the same department. His research interests include pattern recognition, neural networks, evolutionary computation, image and signal processing and robotics. He is a member of IEEE.

Satoru Tsuge (Member) received the B.E., M.E. and D.E.

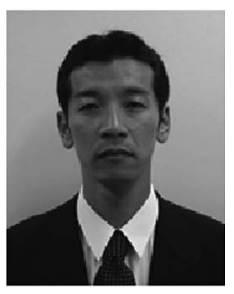
degrees in Engineering from the University of Tokushima, Japan, in 1996, 1998, and 2001 respectively. In 2001, he became an Assistant Professor in the Faculty of Engineering at the University of Tokushima. Until 2010, he was a Lecturer in the Institute of Technology and Science at the University of Tokushima. Currently, he is an Associate Professor at Daido University. He is engaged in research on speech recognition and information retrieval. He is a member of IEEJ, IPSJ and ASJ.

Minoru Fukumi (Member) received the B.E. and M.E. de-

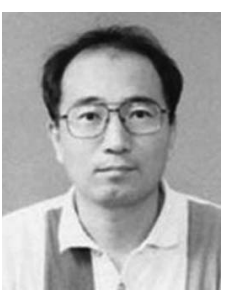
grees from the University of Tokushima, in 1984 and 1987, respectively, and the Doctor Degree from Kyoto University in 1996. Since 1987, he has been with the Department of Information Science and Intelligent Systems, University of Tokushima. In 2005, he became a Professor in the same department. He received the best paper award from the SICE in 1995 and best paper awards from several international conferences. His research interests include neural networks, evolutionary algorithms, image processing and human sensing. He is a member of the IEEE, SICE, IPSJ and IEICE. 